\title{
An improved correlation coefficient between intuitionistic fuzzy sets and its applications to real-life decision-making problems
}

\author{
Paul Augustine Ejegwa \\ Department of Mathematics/Statistics/Computer Science \\ University of Agriculture, P. M. B. 2373, Makurdi, Nigeria \\ e-mail: ejegwa. augustine@uam.edu.ng
}

Received: 15 June 2020

Revised: 15 August 2020

Accepted: 19 August 2020

\begin{abstract}
Correlation coefficient between intuitionistic fuzzy sets (CCIFSs) is a vital research area in intuitionistic fuzzy set theory and has great practical application in a variety of areas. Many methods of computing CCIFSs have been studied hitherto. Due to the weakness in some existing methods of computing CCIFSs, an advanced CCIFSs technique is proposed in this paper which has some advantages over the similar existing methods. This new CCIFSs technique is an improved version of some CCIFSs techniques. A set of numerical illustrations are given to determine the effectiveness of the introduced CCIFSs method over the similar existing ones. Furthermore, we apply the new technique of computing CCIFSs to solve real-life decisionmaking (RLDM) problems of personnel appointment exercise and career determination problem represented in intuitionistic fuzzy values. This proposed measuring tool could be exploited in other multi-criteria decision-making problems via cluster algorithm approach.
\end{abstract}

Keywords: Intuitionistic fuzzy set, Correlation coefficient measure, Real-life decision-making. 2010 Mathematics Subject Classification: 03E72, 62H20, 62M10.

\section{Introduction}

Decision making is a global process in real-life situations, which can be described as the final consequence of some reasoning and logical processes leading to the selection of the best alternative/choice. In some circumstances, it is challenging for decision makers to accurately 
express a preference regarding relevant choices under several criteria, especially when relying on imprecise, indeterminate, or imperfect information. To this end, the theory of fuzzy sets is introduced by Zadeh [34] to address real-life decision-making (RLDM) problems in the presence of uncertainties. The fuzzy value/number is commonly used by the decision maker to express preference of a choice with respect to a certain criterion, which means the degree to which the choice satisfies the criterion.

Roughly two decades after the introduction of fuzzy sets, Atanassov [1,2] showed that in quite a lot of RLDM problems the decision makers may not only provide the grade to which the alternate satisfies the criterion but also give the grade to which the alternate dissatisfies the criterion and so introduced the concept of intuitionistic fuzzy set (IFS) which is an extension of fuzzy sets and characterized by membership and non-membership degreee satisfying the condition that their sum is equal to or less than one. IFS assigns to each element a membership degree and a non-membership degree with a hesitation degree such that the sum of the three parameters is one. Hence, the IFSs are very interesting and give us the possibility to model hesitation and uncertainty by using an additional degree unlike in fuzzy set, where only membership degree is considered and the non-membership degree equals one minus the membership degree. IFSs have been applied in many applicative areas like medical diagnosis, engineering, pattern recognition, career determination, machine learning, logical programming and so on. The following articles contain an amazing applications of IFSs in real-life problems $[3,4,6,9,11-15,21,25,26,30,31]$.

The concept of correlation analysis has been studied in fuzzy sets due to its advantage in decision-making $[5,7,8]$. Correlation coefficient for IFSs is an important research area in IFS theory, and so has received much response from researchers [17-20, 24, 27-29, 32, 33]. Gerstenkorn and Manko [17] defined a function measuring the correlation of IFSs, and introduced a correlation coefficient and examined its properties. Hong and Hwang [18] proposed the concepts of CCIFSs in probability finite spaces. Hung [19] studied a method to calculate the CCIFSs from statistical viewpoint. Hung and $\mathrm{Wu}$ [20] proposed a method to calculate the CCIFSs by means of centroid. Liu et al. [23] studied a new approach to measuring the correlation degree between the IFSs in finite sets capturing membership degree and the non-membership degree of IFSs. Ejegwa [10] proposed a novel CCIFSs method that corrected and generalized a method of CCIFSs studied in [33]. In this paper, the technique of CCIFSs in [10] is improved upon for a better performance, and applied to RLDM problems of personnel appointment exercise and career determination problem represented in intuitionistic fuzzy values. The objectives of the paper are to

(i) reiterate some approaches of calculating CCIFSs in $[10,16,33]$ and propose a new CCIFSs technique that shows accuracy and reliability in measuring CCIFSs.

(ii) mathematically substantiate the proposed CCIFSs technique, and numerically verify the authenticity of the new technique over the methods in $[10,16,33]$.

(iii) demonstrate the application of the new technique in RLDM problems of personnel appointment exercise and career determination. 
To expedite the discussion, the remaining parts of this paper are structured as follows: In Section 2, some fundamental of IFSs are briefly reviewed. In Section 3, some similar existing CCIFSs methods are reviewed and the new CCIFSs technique is introduced and characterized. It also discusses some numerical verifications to validate the proposed approach along with comparative study. In Section 4, we present the applications of the new method in personnel appointments and career determination. Finally, the paper ends with a concluding remark in Section 5 .

\section{Preliminaries}

Brief fundamentals of IFSs for reference and completeness are recalled. Suppose $S$ is a non-empty set that is fixed, then the following definitions follow.

Definition 2.1 ( [34]). A fuzzy set $\tilde{E}$ of $S$ which is characterized by a membership function $\mu_{\tilde{E}}: S \rightarrow[0,1]$ is of the form

$$
\tilde{E}=\left\{\left\langle s, \mu_{\tilde{E}}(s)\right\rangle \mid s \in S\right\} .
$$

Definition 2.2 ([1]). An IFS $E$ of $S$ is an object having the form

$$
E=\left\{\left\langle\frac{\mu_{E}(s), \nu_{E}(s)}{s}\right\rangle \mid s \in S\right\}
$$

or

$$
E=\left\{\left\langle s, \mu_{E}(s), \nu_{E}(s)\right\rangle \mid s \in S\right\}
$$

where the functions

$$
\mu_{E}(s): S \rightarrow[0,1] \text { and } \nu_{E}(s): S \rightarrow[0,1]
$$

are the degree of membership and the degree of non-membership, respectively of the element $s \in S$ to $E$, and for every $s \in S$,

$$
0 \leq \mu_{E}(s)+\nu_{E}(s) \leq 1
$$

For each $E$ of $S$,

$$
\pi_{E}(s)=1-\mu_{E}(s)-\nu_{E}(s)
$$

is the intuitionistic fuzzy set index or hesitation margin of $s$ in $S$. The hesitation margin $\pi_{E}(s)$ is the degree of non-determinacy of $s \in S$, to $E$ and $\pi_{E}(s) \in[0,1]$. The hesitation margin is the function that states lack of knowledge of whether $s \in S$ or $s \notin S$. Thus,

$$
\mu_{E}(s)+\nu_{E}(s)+\pi_{E}(s)=1 \text {. }
$$

Example 2.1. Let $S=\left\{s_{1}, s_{2}, s_{3}\right\}$ be a fixed universe of discourse and

$$
E=\left\{\left\langle\frac{0.7,0.2}{s_{1}}\right\rangle,\left\langle\frac{0.5,0.3}{s_{2}}\right\rangle,\left\langle\frac{0.8,0.2}{s_{3}}\right\rangle\right\}
$$

be an intuitionistic fuzzy set of $S$. Then, the indexes of the elements $s_{1}, s_{2}, s_{3}$ to $E$ are

$$
\pi_{E}\left(s_{1}\right)=0.1, \pi_{E}\left(s_{2}\right)=0.2 \text { and } \pi_{E}\left(s_{3}\right)=0.0 .
$$


Definition 2.3 ([2]). Suppose $E, F \in \operatorname{IFS}(S)$, where $\operatorname{IFS}(S)$ denotes the set of all IFSs of $S$. Then, we have the following:

(i) $\bar{E}=\left\{\left\langle s, \nu_{E}(s), \mu_{E}(s)\right\rangle \mid s \in S\right\}$.

(ii) $E \cup F=\left\{\left\langle s, \max \left(\mu_{E}(s), \mu_{F}(s)\right), \min \left(\nu_{E}(s), \nu_{F}(s)\right)\right\rangle \mid x \in S\right\}$.

(iii) $E \cap F=\left\{\left\langle s, \min \left(\mu_{E}(s), \mu_{F}(s)\right), \max \left(\nu_{E}(s), \nu_{F}(s)\right)\right\rangle \mid s \in S\right\}$.

(iv) $E \oplus F=\left\{\left\langle s, \mu_{E}(s)+\mu_{F}(s)-\mu_{E}(s) \mu_{F}(s), \nu_{E}(s) \nu_{F}(s)\right\rangle \mid s \in S\right\}$.

(v) $E \otimes F=\left\{\left\langle s, \mu_{E}(s) \mu_{F}(s), \nu_{E}(s)+\nu_{F}(s)-\nu_{E}(s) \nu_{F}(s)\right\rangle \mid s \in S\right\}$.

Definition 2.4 ([2]). Let $E$ and $F$ be IFSs of $S$. Then,

$$
E=F \Leftrightarrow \mu_{E}(s)=\mu_{F}(s) \text { and } \nu_{E}(s)=\nu_{F}(s) \forall s \in S,
$$

and

$$
E \subseteq F \Leftrightarrow \mu_{E}(s) \leq \mu_{F}(s) \text { and } \nu_{E}(s) \geq \nu_{F}(s) \forall s \in S .
$$

We say $E \subset F \Leftrightarrow E \subseteq F$ and $E \neq F$. Also, $E$ and $F$ are comparable to each other if $E \subseteq F$ and $F \subseteq E$.

Definition 2.5 ([2]). Suppose $E \in I F S(S)$. Then, the level/ground set or support of $E$ is defined by

$$
E_{*}=\left\{s \in S \mid \mu_{E}(s)>0, \nu_{E}(s)<1\right\},
$$

and the set $E^{*}$ is defined by

$$
E^{*}=\left\{s \in S \mid \mu_{E}(s) \geq 0, \nu_{E}(s) \leq 1\right\} .
$$

Certainly, $E_{*}$ and $E^{*}$ are subsets of $S$.

Definition 2.6 ( [10]). Intuitionistic fuzzy pairs (IFPs) or intuitionistic fuzzy values (IFVs) is an object in the form $\langle x, y\rangle$, where $x, y \in[0,1]$, and $x+y \leq 1$. IFPs are used for the evaluation of objects or processes and which components ( $x$ and $y$ ) are interpreted as degrees of membership and non-membership or degrees of validity and non-validity or degrees of correctness and non-correctness.

\section{Correlation coefficient between intuitionistic fuzzy sets}

In this section, some existing similar/triparametric methods of computing CCIFSs are revisited. Subsequently, the new triparametric CCIFSs technique that improved the method in [10] is given. A comparative analysis of the similar existing CCIFSs methods and the new CCIFSs method is carried out to ascertain the reliability of the new CCIFSs method. Firstly, the axiomatic definition of CCIFSs is given. 
Definition 3.1 ([17]). Let $E$ and $F$ be IFSs of a nonempty set $S$. Then, the correlation coefficient denoted by $\mathcal{K}(E, F)$ is a measuring function $\mathcal{K}: \operatorname{IFS} \times \operatorname{IFS} \rightarrow[0,1]$ satisfying the following axioms;

(i) $\mathcal{K}(E, F) \in[0,1]$,

(ii) $\mathcal{K}(E, F)=\mathcal{K}(F, E)$,

(iii) $\mathcal{K}(E, F)=1$ if and only if $E=F$.

\subsection{Xu et al.'s CCIFSs method}

By modifying the method in [17], Xu et al. [33] proposed the following correlation coefficient for IFSs:

Definition 3.2 ([33]). Let $E$ and $F$ be IFSs of a nonempty set $S=\left\{s_{1}, \ldots, s_{n}\right\}$. Then, the correlation coefficient between $E$ and $F$ is

$$
\mathbf{K}(E, F)=\frac{\mathcal{C}(E, F)}{\max [\sqrt{\mathcal{T}(E)}, \sqrt{\mathcal{T}(F)}]}
$$

where $\mathcal{C}(E, F)$ is the correlation of IFSs, $\mathcal{T}(E)$ and $\mathcal{T}(F)$ are informational energies of $E$ and $F$ respectively, defined as follows:

$$
\begin{gathered}
\mathcal{C}(E, F)=\sum_{i=1}^{n}\left[\mu_{E}\left(s_{i}\right) \mu_{F}\left(s_{i}\right)+\nu_{E}\left(s_{i}\right) \nu_{F}\left(s_{i}\right)+\pi_{E}\left(s_{i}\right) \pi_{F}\left(s_{i}\right)\right], \\
\mathcal{T}(E)=\sum_{i=1}^{n}\left[\mu_{E}^{2}\left(s_{i}\right)+\nu_{E}^{2}\left(s_{i}\right)+\pi_{E}^{2}\left(s_{i}\right)\right]
\end{gathered}
$$

and

$$
\mathcal{T}(F)=\sum_{i=1}^{n}\left[\mu_{F}^{2}\left(s_{i}\right)+\nu_{F}^{2}\left(s_{i}\right)+\pi_{F}^{2}\left(s_{i}\right)\right] .
$$

\subsubsection{Limitation of Xu et al.'s CCIFSs method}

The CCIFSs method in [33] is not an appropriate CCIFSs tool because it violates condition (iii) in Definition 3.1. If $E=F$, then Xu et al. [33]'s correlation coefficient becomes

$$
\begin{aligned}
\mathbf{K}(E, F) & =\frac{\mathcal{C}(E, E)}{\max [\sqrt{\mathcal{T}(E)}, \sqrt{\mathcal{T}(E)}]} \\
& =\frac{\mathcal{T}(E)}{\sqrt{\mathcal{T}(E)}}=\sqrt{\mathcal{T}(E)} \neq 1 .
\end{aligned}
$$

Thus, $\mathbf{K}$ is not reliable. 


\subsection{Garg's correlation coefficients in intuitionistic fuzzy environment}

Definition 3.3 ([16]). Let $E$ and $F$ be IFSs of a nonempty set $S=\left\{s_{1}, \ldots, s_{n}\right\}$. Then, the correlation coefficient between $E$ and $F$ is

$$
\mathcal{K}_{1}(E, F)=\frac{\mathcal{C}(E, F)}{\max [\mathcal{T}(E), \mathcal{T}(F)]},
$$

where

$$
\begin{gathered}
\mathcal{C}(E, F)=\sum_{i=1}^{n}\left[\mu_{E}^{2}\left(s_{i}\right) \mu_{F}^{2}\left(s_{i}\right)+\nu_{E}^{2}\left(s_{i}\right) \nu_{F}^{2}\left(s_{i}\right)+\pi_{E}^{2}\left(s_{i}\right) \pi_{F}^{2}\left(s_{i}\right)\right], \\
\mathcal{T}(E)=\sum_{i=1}^{n}\left[\mu_{E}^{4}\left(s_{i}\right)+\nu_{E}^{4}\left(s_{i}\right)+\pi_{E}^{4}\left(s_{i}\right)\right]
\end{gathered}
$$

and

$$
\mathcal{T}(F)=\sum_{i=1}^{n}\left[\mu_{F}^{4}\left(s_{i}\right)+\nu_{F}^{4}\left(s_{i}\right)+\pi_{F}^{4}\left(s_{i}\right)\right] .
$$

\subsection{Ejegwa's CCIFSs method}

Definition 3.4 ([10]). Let $E$ and $F$ be IFSs of $S=\left\{s_{1}, \ldots, s_{n}\right\}$. Then, the correlation coefficient measure for $E$ and $F$ is

$$
\mathcal{K}_{2}(E, F)=\frac{\mathcal{C}(E, F)}{\max [\mathcal{T}(E), \mathcal{T}(F)]},
$$

where $\mathcal{C}(E, F), \mathcal{T}(E)$ and $\mathcal{T}(F)$ are informational energies of $E$ and $F$, and correlation between $(E, F)$ are defined by

$$
\begin{gathered}
\mathcal{T}(E)=\sum_{i=1}^{n}\left[\mu_{E}^{k}\left(s_{i}\right)+\nu_{E}^{k}\left(s_{i}\right)+\pi_{E}^{k}\left(s_{i}\right)\right], \\
\mathcal{T}(F)=\sum_{i=1}^{n}\left[\mu_{F}^{k}\left(s_{i}\right)+\nu_{F}^{k}\left(s_{i}\right)+\pi_{F}^{k}\left(s_{i}\right)\right], \\
\mathcal{C}(E, F)=\sum_{i=1}^{n}\left[\mu_{E}^{\frac{k}{2}}\left(s_{i}\right) \mu_{F}^{\frac{k}{2}}\left(s_{i}\right)+\nu_{E}^{\frac{k}{2}}\left(s_{i}\right) \nu_{F}^{\frac{k}{2}}\left(s_{i}\right)+\pi_{E}^{\frac{k}{2}}\left(s_{i}\right) \pi_{F}^{\frac{k}{2}}\left(s_{i}\right)\right],
\end{gathered}
$$

where $k=2 n-1$ for $n=1,2$.

This CCIFSs method satisfies condition (iii) in Definition 3.1 because

$$
\mathcal{K}_{2}(E, F)=\frac{\mathcal{C}(E, E)}{\max [\mathcal{T}(E), \mathcal{T}(E)]}=\frac{\mathcal{T}(E)}{\mathcal{T}(E)}=1
$$

Hence, $\mathcal{K}_{2}$ is a reliable correlation coefficient for IFSs. However, for a better output, we propose an improved version of Eq. (16), as follows. 


\subsection{New CCIFSs technique}

Definition 3.5. Let $E$ and $F$ be IFSs of $S=\left\{s_{1}, s_{2}, \ldots, s_{n}\right\}$. Then, the new correlation coefficient measure for $E$ and $F$ is

$$
\mathcal{K}_{3}(E, F)=\frac{\mathcal{C}(E, F)}{\operatorname{Aver}[\mathcal{T}(E), \mathcal{T}(F)]},
$$

where $\mathcal{C}(E, F), \mathcal{T}(E)$ and $\mathcal{T}(F)$ are as given in Eqs. (17), (18) and (19).

Thus, Eq. (20) could also be written as

$$
\mathcal{K}_{3}(E, F)=\frac{\mathcal{C}(E, F)}{\operatorname{Aver}[\mathcal{C}(E, E), \mathcal{C}(F, F)]} .
$$

Remark 3.1. Suppose $E$ and $F$ are IFSs of $S=\left\{s_{1}, s_{2}, \ldots, s_{n}\right\}$. Then, $\mathcal{K}_{2}(E, F)$ and $\mathcal{K}_{3}(E, F)$ are equal if and only if $\mathcal{T}(E)=\mathcal{T}(F)$.

Theorem 3.1. Suppose $E$ and $F$ are IFSs of $S$. Then, the function $\mathcal{K}_{3}(E, F)$ is a correlation coefficient of $E$ and $F$.

Proof. To show that $\mathcal{K}_{3}(E, F)$ is a CCIFSs $E$ and $F$, we verify the conditions in Definition 3.1. Firstly, we show that $\mathcal{K}_{3}(E, F) \in[0,1]$, which implies $0 \leq \mathcal{K}_{3}(E, F) \leq 1$. But $\mathcal{K}_{3}(E, F) \geq 0$ is trivial since $\mathcal{C}(E, F) \geq 0$ and $[\mathcal{T}(E), \mathcal{T}(F)]>0$.

To show that $\mathcal{K}_{3}(E, F) \leq 1$, we make the following assumptions, i.e., let

$$
\begin{array}{ll}
\sum_{i=1}^{n} \mu_{E}^{k}\left(x_{i}\right)=a, & \sum_{i=1}^{n} \mu_{F}^{k}\left(x_{i}\right)=b, \\
\sum_{i=1}^{n} \nu_{E}^{k}\left(x_{i}\right)=c, & \sum_{i=1}^{n} \nu_{F}^{k}\left(x_{i}\right)=d, \\
\sum_{i=1}^{n} \pi_{E}^{k}\left(x_{i}\right)=e, & \sum_{i=1}^{n} \pi_{F}^{k}\left(x_{i}\right)=f .
\end{array}
$$

Recall that $\mathcal{K}_{3}(E, F)=\frac{\mathcal{C}(E, F)}{\operatorname{Aver}[\mathcal{T}(E), \mathcal{T}(F)]}$. Applying the principle of Cauchy-Schwarz's inequality, we have

$$
\begin{aligned}
\mathcal{K}_{3}(E, F) & =\frac{\sum_{i=1}^{n}\left[\mu_{E}^{\frac{k}{2}}\left(x_{i}\right) \mu_{F}^{\frac{k}{2}}\left(x_{i}\right)+\nu_{E}^{\frac{k}{2}}\left(x_{i}\right) \nu_{F}^{\frac{k}{2}}\left(x_{i}\right)+\pi_{E}^{\frac{k}{2}}\left(x_{i}\right) \pi_{F}^{\frac{k}{2}}\left(x_{i}\right)\right]}{A \operatorname{ver}\left[\sum_{i=1}^{n}\left(\mu_{E}^{k}\left(x_{i}\right)+\nu_{E}^{k}\left(x_{i}\right)+\pi_{E}^{k}\left(x_{i}\right)\right), \sum_{i=1}^{n}\left(\mu_{F}^{k}\left(x_{i}\right)+\nu_{F}^{k}\left(x_{i}\right)+\pi_{F}^{k}\left(x_{i}\right)\right)\right]} \\
& =\frac{\sum_{i=1}^{n} \mu_{E}^{\frac{k}{2}}\left(x_{i}\right) \mu_{F}^{\frac{k}{2}}\left(x_{i}\right)+\sum_{i=1}^{n} \nu_{E}^{\frac{k}{2}}\left(x_{i}\right) \nu_{F}^{\frac{k}{2}}\left(x_{i}\right)+\sum_{i=1}^{n} \pi_{E}^{\frac{k}{2}}\left(x_{i}\right) \pi_{F}^{\frac{k}{2}}\left(x_{i}\right)}{A \operatorname{ver}\left[\left(\sum_{i=1}^{n} \mu_{E}^{k}\left(x_{i}\right)+\sum_{i=1}^{n} \nu_{E}^{k}\left(x_{i}\right)+\sum_{i=1}^{n} \pi_{E}^{k}\left(x_{i}\right)\right),\left(\sum_{i=1}^{n} \mu_{F}^{k}\left(x_{i}\right)+\sum_{i=1}^{n} \nu_{F}^{k}\left(x_{i}\right)+\sum_{i=1}^{n} \pi_{F}^{k}\left(x_{i}\right)\right)\right]} \\
& \leq \frac{\left[\sum_{i=1}^{n} \mu_{E}^{k}\left(x_{i}\right) \sum_{i=1}^{n} \mu_{F}^{k}\left(x_{i}\right)\right]^{\frac{1}{2}}+\left[\sum_{i=1}^{n} \nu_{E}^{k}\left(x_{i}\right) \sum_{i=1}^{n} \nu_{F}^{k}\left(x_{i}\right)\right]^{\frac{1}{2}}+\left[\sum_{i=1}^{n} \pi_{E}^{k}\left(x_{i}\right) \sum_{i=1}^{n} \pi_{F}^{k}\left(x_{i}\right)\right]^{\frac{1}{2}}}{A \operatorname{ver}\left[\left(\sum_{i=1}^{n} \mu_{E}^{k}\left(x_{i}\right)+\sum_{i=1}^{n} \nu_{E}^{k}\left(x_{i}\right)+\sum_{i=1}^{n} \pi_{E}^{k}\left(x_{i}\right)\right),\left(\sum_{i=1}^{n} \mu_{F}^{k}\left(x_{i}\right)+\sum_{i=1}^{n} \nu_{F}^{k}\left(x_{i}\right)+\sum_{i=1}^{n} \pi_{F}^{k}\left(x_{i}\right)\right)\right.} \\
& =\frac{(a b)^{\frac{1}{2}}+(c d)^{\frac{1}{2}}+(e f)^{\frac{1}{2}}}{A \operatorname{ver}[(a+c+e),(b+d+f)]}
\end{aligned}
$$


But,

$$
\begin{aligned}
\mathcal{K}_{3}(E, F)-1 & \leq \frac{(a b)^{\frac{1}{2}}+(c d)^{\frac{1}{2}}+(e f)^{\frac{1}{2}}}{A \operatorname{ver}[(a+c+e),(b+d+f)]}-1 \\
& =\frac{(a b)^{\frac{1}{2}}+(c d)^{\frac{1}{2}}+(e f)^{\frac{1}{2}}-A \operatorname{err}[(a+c+e),(b+d+f)]}{A \operatorname{ver}[(a+c+e),(b+d+f)]} \\
& =\frac{-\left\{A \operatorname{ver}[(a+c+e),(b+d+f)]-\left[(a b)^{\frac{1}{2}}+(c d)^{\frac{1}{2}}+(e f)^{\frac{1}{2}}\right]\right\}}{A v e r[(a+c+e),(b+d+f)]} \\
& =-\frac{\left\{A \operatorname{ver}[(a+c+e),(b+d+f)]-\left[(a b)^{\frac{1}{2}}+(c d)^{\frac{1}{2}}+(e f)^{\frac{1}{2}}\right]\right\}}{A \operatorname{ver}[(a+c+e),(b+d+f)]} \\
& \leq 0 .
\end{aligned}
$$

Thus, $\mathcal{K}_{3}(E, F) \leq 1$. Hence, condition (i) holds.

Certainly, $\mathcal{K}(E, F)=\mathcal{K}(F, E)$. Hence, condition (ii) holds. Again, $\mathcal{K}_{3}(E, F)=1 \Leftrightarrow E=F$ $\Rightarrow$

$$
\mathcal{K}_{3}(E, F)=\frac{\mathcal{C}(E, E)}{\operatorname{Aver}[\mathcal{T}(E), \mathcal{T}(E)]}=\frac{\mathcal{T}(E)}{\mathcal{T}(E)}=1,
$$

This proves condition (iii). Therefore, $\mathcal{K}_{3}(E, F)$ is a correlation coefficient of $E$ and $F$.

\subsection{Numerical illustrations of the new CCIFSs method}

Now, we show the reliability of the new CCIFSs method over the similar existing ones via numerical examples.

\subsubsection{Example I}

Let $E$ and $F$ be IFSs in $S=\left\{s_{1}, s_{2}, s_{3}\right\}$ defined by

$$
E=\left\{\left\langle\frac{0.3,0.6,0.1}{s_{1}}\right\rangle,\left\langle\frac{0.5,0.3,0.2}{s_{2}}\right\rangle,\left\langle\frac{0.4,0.5,0.1}{s_{3}}\right\rangle\right\}
$$

and

$$
F=\left\{\left\langle\frac{0.3,0.6,0.1}{s_{1}}\right\rangle,\left\langle\frac{0.5,0.3162,0.1838}{s_{2}}\right\rangle,\left\langle\frac{0.3873,0.5,0.1127}{s_{3}}\right\rangle\right\} .
$$

We compute the CCIFSs $E$ and $F$ as follows:

Using Eq. (12), we get

$$
\mathcal{K}_{1}(E, F)=0.2120 .
$$

By using Eq. (16), we obtain the following for $k=1$ and $k=3$ :

$$
\mathcal{K}_{2}(E, F)=0.7051 \mathcal{K}_{2}(E, F)=0.2982 .
$$

By using Eq. (20), we have the following for $k=1$ and $k=3$ :

$$
\mathcal{K}_{3}(E, F)=0.7051, \mathcal{K}_{3}(E, F)=0.4828 .
$$




\subsubsection{Example II}

Suppose there are two IFSs $\tilde{E}$ and $\tilde{F}$ of a nonempty set $S=\left\{s_{1}, s_{2}, s_{3}\right\}$ such that

$$
\tilde{E}=\left\{\left\langle\frac{0.1,0.2,0.7}{s_{1}}\right\rangle,\left\langle\frac{0.2,0.1,0.7}{s_{2}}\right\rangle,\left\langle\frac{0.29,0.0,0.71}{s_{3}}\right\rangle\right\}
$$

and

$$
\tilde{F}=\left\{\left\langle\frac{0.1,0.3,0.6}{s_{1}}\right\rangle,\left\langle\frac{0.2,0.2,0.6}{s_{2}}\right\rangle,\left\langle\frac{0.29,0.1,0.61}{s_{3}}\right\rangle\right\} .
$$

Using Eq. (12), we get

$$
\mathcal{K}_{1}(\tilde{E}, \tilde{F})=0.7426
$$

Using Eq. (16), we obtain the following for $k=1$ and $k=3$ :

$$
\mathcal{K}_{2}(\tilde{E}, \tilde{F})=0.9769, \mathcal{K}_{2}(\tilde{E}, \tilde{F})=0.8104
$$

Using Eq. (20), we have the following for $k=1$ and $k=3$ :

$$
\mathcal{K}_{3}(\tilde{E}, \tilde{F})=0.9769, \mathcal{K}_{3}(\tilde{E}, \tilde{F})=0.9701
$$

\begin{tabular}{|l||l|l|}
\hline CCIFSs techniques & Example I & Example II \\
\hline \hline Garg [16] & 0.2120 & 0.7426 \\
Ejegwa [10] for $k=1$ & 0.7051 & 0.9769 \\
Ejegwa [10] for $k=3$ & 0.2982 & 0.8104 \\
New CCIFSs technique for $k=1$ & $\mathbf{0 . 7 0 5 1}$ & $\mathbf{0 . 9 7 6 9}$ \\
New CCIFSs technique for $k=3$ & $\mathbf{0 . 4 8 2 8}$ & $\mathbf{0 . 9 7 0 1}$ \\
\hline
\end{tabular}

Table 1. Numerical Output for Example I

\subsubsection{Discussion}

From Table 1, Garg [16] approach shows that the CCIFSs $(E, F)$ and $(\tilde{E}, \tilde{F})$ are 0.2120 and 0.7426. The CCIFSs method in [10] gives $0.7051,0.2982$ and $0.9769,0.8104$, respectively for $k=1,3$. Whereas that of the proposed method yields $0.7051,0.4828$ and $0.9769,0.9701$, respectively for $k=1,3$. The proposed method gives a better correlation coefficient when compare to the methods in $[10,16]$. Certainly, Eq. (16) gives a better result in comparison to Eq. (12). The correlation coefficient using Eqs. (12) and (16) for $k=1$ is the same because the informational energies of both cases are equal in agreement to Remark 3.1. The correlation coefficient value for the proposed method decreases as $k$ increases and it is very reliable because it has two alternates. That is, whenever one alternate fails to give a reasonable correlation, the another alternate could be employed.

\section{Real-life decision-making problems through the new method}

In this section, some RLDM problems in personnel appointment and career determination are discussed via the new CCIFSs technique. 


\subsection{Applicative example of personnel appointment}

Suppose a company wants to employ a competent officer into a vacant position, $P$ where there are four applicants, $A_{i}$ for $i=1,2,3,4$. The task is to select the most qualified applicant from $A_{i}$ without a mistake, since such will cost the company greatly. Assume the applicants are assessed under the stipulated qualifications, $S=\left\{s_{1}, s_{2}, s_{3}, s_{4}, s_{5}\right\}$, where $s_{1}=$ honesty, $s_{2}=$ team spirit, $s_{3}=$ hardworking, $s_{4}=$ transparency, $s_{5}=$ academic qualification and the scores in IFVs are as follow:

$$
\begin{aligned}
& A_{1}=\left\{\left\langle\frac{0.5,0.1,0.4}{s_{1}}\right\rangle,\left\langle\frac{0.6,0.1,0.3}{s_{2}}\right\rangle,\left\langle\frac{1.0,0.0,0.0}{s_{3}}\right\rangle,\left\langle\frac{0.8,0.1,0.1}{s_{4}}\right\rangle,\left\langle\frac{0.4,0.2,0.4}{s_{5}}\right\rangle\right\} \\
& A_{2}=\left\{\left\langle\frac{0.8,0.1,0.1}{s_{1}}\right\rangle,\left\langle\frac{0.6,0.3,0.1}{s_{2}}\right\rangle,\left\langle\frac{0.7,0.0,0.3}{s_{3}}\right\rangle,\left\langle\frac{0.6,0.2,0.2}{s_{4}}\right\rangle,\left\langle\frac{0.5,0.2,0.3}{s_{5}}\right\rangle\right\} \\
& A_{3}=\left\{\left\langle\frac{0.5,0.4,0.1}{s_{1}}\right\rangle,\left\langle\frac{0.6,0.1,0.3}{s_{2}}\right\rangle,\left\langle\frac{0.5,0.1,0.4}{s_{3}}\right\rangle,\left\langle\frac{0.5,0.2,0.3}{s_{4}}\right\rangle,\left\langle\frac{1.0,0.0,0.0}{s_{5}}\right\rangle\right\} \\
& A_{4}=\left\{\left\langle\frac{0.9,0.1,0.0}{s_{1}}\right\rangle,\left\langle\frac{0.8,0.2,0.0}{s_{2}}\right\rangle,\left\langle\frac{1.0,0.0,0.0}{s_{3}}\right\rangle,\left\langle\frac{1.0,0.0,0.0}{s_{4}}\right\rangle,\left\langle\frac{1.0,0.0,0.0}{s_{5}}\right\rangle\right\} .
\end{aligned}
$$

The company requirements for the position, $P$ in IFVs with respect to the stipulated qualifications is given as

$$
P=\left\{\left\langle\frac{0.9,0.1,0.0}{s_{1}}\right\rangle,\left\langle\frac{1.0,0.0,0.0}{s_{2}}\right\rangle,\left\langle\frac{1.0,0.0,0.0}{s_{3}}\right\rangle,\left\langle\frac{0.8,0.2,0.0}{s_{4}}\right\rangle,\left\langle\frac{1.0,0.0,0.0}{s_{5}}\right\rangle\right\} .
$$

By using the new CCIFSs technique for $k=3$ to compute the correlation coefficient between each applicant and the vacant position, the following results are obtain:

$$
\begin{aligned}
& \mathcal{K}_{3}\left(A_{1}, P\right)=0.8006, \mathcal{K}_{3}\left(A_{2}, P\right)=0.8168, \\
& \mathcal{K}_{3}\left(A_{3}, P\right)=0.7919, \mathcal{K}_{3}\left(A_{4}, P\right)=0.9791 .
\end{aligned}
$$

From the computations, applicant $A_{4}$ is suitable to be employed ahead of all the other applicants because the correlation coefficient between $A_{4}$ and $P$ is the greatest.

\subsection{Applicative example of career determination}

Career determination is a real-life decision-making process where a choice of a career is made for effective performance. In career determination, career assessments are very vital tools. These tools are designed to assist candidates understand how a variation of personal characteristics such interests, values, preferences, motivations, aptitudes, skills, etc. impact their potential achievement with different career choices and work environments. Assessments of some or all of these characteristics are often used by individuals or institutions, such as university career centers, career counsellors, corporate human resources staff, and guidance counsellors among others, to help individuals make more informed career decisions. Career guidance helps high school students determine which subject they may want to concentrate on to reach a chosen career path [22]. Career assessments are intended to discover the skills, aptitudes and talents of candidates 
to enhance choosing a career that is in tune with the candidates' goals and talents. Career assessments, in the form of tests can be useful for those who are indeterminate about the myriad of career opportunities. Factors like academic performance, interest, personality make-up etc., determine career choice and placement but the first mentioned seems to be prominent [12]. However, some issues may affect student's academic performance called test contingencies viz:

(i) student's socio-economic background, health, anxiety, interest, mood, etc.

(ii) teachers' attitudes, instructional techniques, qualification or competency, etc.

(iii) environmental influences like weather condition, time of day, sitting arrangement, invigilation, etc.

(iv) others like appropriateness of the exam format, duration of the exam, appropriateness of the exam objective, relevance and adequacy of the exam to the syllabus, malpractice, marking scheme or teacher's mood when marking, etc.

Suppose four candidates, $C=\left\{C_{1}, C_{2}, C_{3}, C_{4}\right\}$ sat for a University Tertiary Matriculation Examination (UTME) free from test contingencies to compete to study a course, $X$ due to limited opportunity. The candidates sat for five subjects, $S=\left\{s_{1}, s_{2}, s_{3}, s_{4}, s_{5}\right\}$ where $s_{1}=$ English Language, $s_{2}=$ Biology, $s_{3}=$ Mathematics, $s_{4}=$ Chemistry, $s_{5}=$ Physics. Assume the UTME board stipulates the requisites for studying $X$ in IFVs given by

$$
X=\left\{\left\langle\frac{0.9,0.1,0.0}{s_{1}}\right\rangle,\left\langle\frac{1.0,0.0,0.0}{s_{2}}\right\rangle,\left\langle\frac{0.0,1.0,0.0}{s_{3}}\right\rangle,\left\langle\frac{1.0,0.0,0.0}{s_{4}}\right\rangle,\left\langle\frac{0.7,0.1,0.2}{s_{5}}\right\rangle\right\} .
$$

After the candidates sat for the UTME, their performances were represented in IFVs below:

$$
\begin{aligned}
& C_{1}=\left\{\left\langle\frac{0.6,0.3,0.1}{s_{1}}\right\rangle,\left\langle\frac{0.5,0.4,0.1}{s_{2}}\right\rangle,\left\langle\frac{0.6,0.4,0.0}{s_{3}}\right\rangle,\left\langle\frac{0.6,0.2,0.2}{s_{4}}\right\rangle,\left\langle\frac{0.5,0.5,0.0}{s_{5}}\right\rangle\right\}, \\
& C_{2}=\left\{\left\langle\frac{0.5,0.3,0.2}{s_{1}}\right\rangle,\left\langle\frac{0.9,0.1,0.0}{s_{2}}\right\rangle,\left\langle\frac{0.3,0.4,0.3}{s_{3}}\right\rangle,\left\langle\frac{0.9,0.0,0.1}{s_{4}}\right\rangle,\left\langle\frac{0.2,0.7,0.1}{s_{5}}\right\rangle\right\}, \\
& C_{3}=\left\{\left\langle\frac{0.7,0.1,0.2}{s_{1}}\right\rangle,\left\langle\frac{0.6,0.3,0.1}{s_{2}}\right\rangle,\left\langle\frac{0.7,0.1,0.2}{s_{3}}\right\rangle,\left\langle\frac{0.5,0.4,0.1}{s_{4}}\right\rangle,\left\langle\frac{0.4,0.5,0.1}{s_{5}}\right\rangle\right\}, \\
& C_{4}=\left\{\left\langle\frac{1.0,0.0,0.0}{s_{1}}\right\rangle,\left\langle\frac{0.9,0.0,0.1}{s_{2}}\right\rangle,\left\langle\frac{0.6,0.0,0.4}{s_{3}}\right\rangle,\left\langle\frac{0.6,0.1,0.3}{s_{4}}\right\rangle,\left\langle\frac{0.2,0.5,0.3}{s_{5}}\right\rangle\right\} .
\end{aligned}
$$

The challenge is to determine which of the candidate is best suitable to study course, $X$ with respect to academic performance via correlation coefficient approach between each of the candidates, $C_{i}$ (for $i=1,2,3,4$ ) and the course, $X$.

By using the new CCIFSs technique for $k=3$ to compute the correlation coefficient between each candidate and the course, we get results below:

$$
\mathcal{K}_{3}\left(C_{1}, X\right)=0.6410, \mathcal{K}_{3}\left(C_{2}, X\right)=0.7587
$$




$$
\mathcal{K}_{3}\left(C_{3}, X\right)=0.5594, \mathcal{K}_{3}\left(C_{4}, X\right)=0.6929 .
$$

From the computational results, it is inferred that the candidate $C_{2}$ is appropriate to study course, $X$ ahead of all the other candidates since

$$
\mathcal{K}_{3}\left(C_{2}, X\right)>\mathcal{K}_{3}\left(C_{4}, X\right)>\mathcal{K}_{3}\left(C_{1}, X\right)>\mathcal{K}_{3}\left(C_{3}, X\right)
$$

\section{Conclusion}

This paper considered a novel approach of finding CCIFSs different from the maximum approach studied in $[10,16]$. The new CCIFSs technique is an improvement of the method in [10]. We mathematically proved that the new CCIFSs technique satisfied the axiomatic conditions of CCIFSs, and it was numerically verified that the proposed method yields a reasonable output in contrast to the methods in $[10,16]$. However, the new CCIFSs technique yields an output which equals the output of the approach in [10] if and only if the informational energies of the intuitionistic fuzzy sets are the same. In an attempt to demonstrate the usefulness of the proposed method, some RLDM problems such as personnel appointment exercise and career determination via academic performance were discussed in details in the framework of IFSs. Hence, it is meet to say that the new CCIFSs method is an appropriate approach for solving RLDM problems efficiently. Exploiting this new CCIFSs method via clustering algorithm could be an interesting area for future research.

\section{References}

[1] Atanassov, K. T. (1986). Intuitionistic fuzzy sets, Fuzzy Sets and Systems, 20 (1), 87-96.

[2] Atanassov, K. T. (1994). New operations defined on intuitionistic fuzzy sets, Fuzzy Sets and Systems, 61, 137-142.

[3] Atanassov, K. T. (1999). Intuitionistic Fuzzy Sets: Theory and Applications. Physica-Verlag, Heidelberg.

[4] Atanassov, K. T. (2012). On Intuitionistic Fuzzy Sets Theory. Springer, Berlin.

[5] Chiang, D. A., \& Lin, N.P. (1999). Correlation of fuzzy sets, Fuzzy Sets and Systems, 102 (2), 221-226.

[6] De, S. K., Biswas, R., \& Roy, A. R. (2001). An application of intuitionistic fuzzy sets in medical diagnosis, Fuzzy Sets and Systems, 117 (2), 209-213.

[7] Dumitrescu, D. (1977). A definition of an informational energy in fuzzy set theory, Studia University Babes-Bolyai Mathematics, 22, 57-59.

[8] Dumitrescu, D. (1978). Fuzzy correlation, Studia University Babes-Bolyai Mathematics, 23, 41-44. 
[9] Ejegwa, P. A. (2015). Intuitionistic fuzzy sets approach in appointment of positions in an organization via max-min-max rule, Global Journal Science Frontier Research: Mathematics and Decision Science, 15 (6), 1-6.

[10] Ejegwa, P. A. (2020). Modified and generalized correlation coefficient between intuitionistic fuzzy sets with applications, Notes on Intuitionistic Fuzzy Sets, 26 (1), 8-22.

[11] Ejegwa, P. A., \& Adamu, I. M. (2019). Distances between intuitionistic fuzzy sets of second type with application to diagnostic medicine, Notes on Intuitionistic Fuzzy Sets, 25 (3), 53-70.

[12] Ejegwa, P. A., Akubo, A. J., \& Joshua, O. M. (2014). Intuitionistic fuzzy set and its application in career determination via normalized Euclidean distance method, European Scientific Journal, 10 (15), 529-536.

[13] Ejegwa, P. A., \& Modom, E. S. (2015). Diagnosis of viral hepatitis using new distance measure of intuitionistic fuzzy sets, International Journal of Fuzzy Mathematical Archive, $8(1), 1-7$.

[14] Ejegwa, P. A., \& Onasanya, B. O. (2019). Improved intuitionistic fuzzy composite relation and its application to medical diagnostic process, Notes on Intuitionistic Fuzzy Sets, 25 (1), 43-58.

[15] Ejegwa, P. A., \& Onyeke, I. C. (2019). An object oriented approach to the application of intuitionistic fuzzy sets in competency based test evaluation, Annals of Communications in Mathematics, 1 (1), 38-47.

[16] Garg, H. (2016). A novel correlation coefficients between Pythagorean fuzzy sets and its applications to decision making processes, International Journal of Intelligent and Systems, 31 (12), 1234-1252.

[17] Gerstenkorn, T., \& Manko, J. (1991). Correlation of intuitionistic fuzzy sets, Fuzzy Sets and Systems, 44 (1), 39-43.

[18] Hong, D. H., \& Hwang, S. Y. (1995). Correlation of intuitionistic fuzzy sets in probability spaces, Fuzzy Sets and Systems, 75, 77-81.

[19] Hung, W. L. (2001). Using statistical viewpoint in developing correlation of intuitionistic fuzzy sets, International Journal of Uncertainty, Fuzziness and Knowledge-Based Systems, 9 (4), 509-516.

[20] Hung, W. L., \& Wu, J. W. (2002). Correlation of intuitionistic fuzzy sets by centroid method, Information Sciences, 144 (1), 219-225.

[21] Iqbal, M. N., \& Rizwan, U. (2019). Some applications of intuitionistic fuzzy sets using new similarity measure, Journal of Ambient Intelligent and Humanized Computing, https: //doi.org/10.1007/s12652-019-01516-7. 
[22] Kapes, J. T., Mastie, M. M., \& Whitfield, E. A. (1994). A Counsellor's Guide to Career Assessment Instruments, Alexandria, VA: National Career Development Association.

[23] Liu, B., Shen, Y., Mu, L., Chen, X., \& Chen, L. (2016). A new correlation measure of the intuitionistic fuzzy sets, Journal of Intelligent and Fuzzy Systems, 30 (2), 1019-1028.

[24] Mitchell, H. B. (2004). A correlation coefficient for intuitionistic fuzzy sets, International Journal of Intelligent and Systems, 19 (5), 483-490.

[25] Szmidt, E., \& Kacprzyk, J. (2001). Intuitionistic fuzzy sets in some medical applications, Notes on Intuitionistic Fuzzy Sets, 7 (4),58-64.

[26] Szmidt, E., \& Kacprzyk, J. (2004). Medical diagnostic reasoning using a similarity measure for intuitionistic fuzzy sets, Notes on Intuitionistic Fuzzy Sets, 10 (4), 61-69.

[27] Szmidt, E., \& Kacprzyk, J. (2010). Correlation of intuitionistic fuzzy sets, Lecture Notes on Computing Science, 6178, 169-177.

[28] Thao, N. X. (2018). A new correlation coefficient of the intuitionistic fuzzy sets and its application, Journal of Intelligent and Fuzzy Systems, 35 (2), 1959-1968.

[29] Thao, N. X., Ali, M., \& Smarandache, F. (2019). An intuitionistic fuzzy clustering algorithm based on a new correlation coefficient with application in medical diagnosis, Journal of Intelligent and Fuzzy Systems, 36 (1), 189-198.

[30] Todorova, L., Atanassov, K. T., Hadjitodorov, S., \& Vassilev, P. (2007). On an intuitionistic fuzzy approach for decision-making in medicine (Part 1), International Electronic Journal of Bioautomation, 6, 92-101.

[31] Todorova, L., Atanassov, K. T., Hadjitodorov, S., \& Vassilev, P. (2007). On an intuitionistic fuzzy approach for decision-making in medicine (Part 2), International Electronic Journal of Bioautomation, 7, 64-69.

[32] $\mathrm{Xu}, \mathrm{Z}$. (2006). On correlation measures of intuitionistic fuzzy sets, Lecture Notes on Computing Science, 4224, 16-24.

[33] Xu, S., Chen, J., \& Wu, J. J. (2008). Cluster algorithm for intuitionistic fuzzy sets, Information Sciences, 178, 3775-3790.

[34] Zadeh, L. A. (1965). Fuzzy sets, Information and Control, 8, 338-353. 